\title{
FATORES SOCIODEMOGRAFICOS E COMPORTAMENTAIS ASSOCIADOS À ADIPOSIDADE CORPORAL EM ADULTOS
}

Sociodemographic and behavioral factors associated with body adiposity in adults.

Factores sociodemográficos y conductuales asociados con la adiposidad corporal en adultos.

\section{Desafios}

Artigo Original Original Article Artículo Original

Tainara Pereira de Araujo ${ }^{* 1}$, Elisama Costa Lopes ${ }^{2}$, Fabiane Aparecida Canaan Rezende ${ }^{3}$, Renata Junqueira Pereira ${ }^{3,4}$

${ }^{1}$ Mestranda em Ciências da Saúde pela Universidade Federal do Tocantins. Palmas - TO, Brasil.

${ }^{2}$ Mestre em Ciências da Saúde pela Universidade Federal do Tocantins. Palmas - TO, Brasil.

${ }^{3}$ Docente do curso de Nutrição da Universidade Federal do Tocantins. Palmas - TO, Brasil.

${ }^{4}$ Docente do Programa de Mestrado em Ciências da Saúde da Universidade Federal do Tocantins. Palmas - TO, Brasil.

*Correspondência: Laboratório de Nutrição, Universidade Federal do Tocantins, Av. NS 15, 109 Norte, Palmas, Tocantins, Brasil. CEP:77.010-090.e-mail: renatajunqueira@uft.edu.br.

\section{RESUMO}

A obesidade é uma condição multicausal, caracterizada pelo excesso de peso, resultante do desequilíbrio do balanço energético. O objetivo deste estudo foi avaliar os fatores sociodemográficos e comportamentais associados ao elevado percentual de gordura corporal (GC) e à obesidade abdominal (OA), numa população adulta, servidores da Universidade Federal do Tocantins. Participaram do estudo 226 servidores, os quais foram submetidos a avaliação antropométrica, e responderam a um questionário socioeconômico e comportamental. As prevalências de sobrepeso e obesidade foram $33,09 \%$ e 16,18\%, respectivamente, para mulheres e 36,67\% e 16,67\%, para homens, a OA esteve relacionada à idade em ambos os sexos, a GC diretamente relacionada à idade e escolaridade nos homens. 67,78\% dos homens consumiam bebida alcoólica; $46,32 \%$ das mulheres eram sedentárias. Nos homens, o tabagismo esteve associado ao aumento do perímetro da cintura (PC). As prevalências de sedentarismo e consumo de bebidas alcoólicas foram consideradas elevadas em relação ao preconizado na literatura. Os percentuais de indivíduos em sobrepeso e obesidade foram maiores entre homens. O PC aumentou conforme aumento da idade nas mulheres. A GC aumentou conforme o grau de escolaridade nos homens. O tabagismo associou-se ao PC elevado nos homens, a prática de atividade física não demostrou-se fator de proteção.

Palavras-chave: Adiposidade. Composição Corporal. Obesidade Abdominal.

\section{ABSTRACT}

Obesity is a multi-causal condition, characterized by excess weight, resulting from energy balance imbalance. The objectives of this study was to evaluate the sociodemographic and behavioral factors associated with the high percentage of body fat $(C G)$ and abdominal obesity $(O A)$ in an adult population, servants of the Federal University of Tocantins. 226 servants participated in the study, who underwent anthropometric assessment and answered a socioeconomic and behavioral questionnaire. The prevalence of overweight and obesity was $33.09 \%$ and $16.18 \%$, respectively, for women and $36.67 \%$ and $16.67 \%$, for men, $A O$ was related to age in both sexes, to BF directly related to age and schooling in men. $67.78 \%$ of men consumed alcoholic beverages; $46.32 \%$ of the women were sedentary. In men, smoking was associated with increased waist circumference (WC). The prevalence of sedentarism and consumption of alcoholic beverages were considered high in relation to that recommended in the literature. The percentages of individuals in overweight and obesity were higher among men. WC increased with increasing age in 
women. The BF increased according to the level of schooling in men. Smoking was associated with elevated WC in men; physical activity practice was not a protective factor.

Keywords: Adiposity. Body Compositio. Abdominal obesity.

\section{RESUMEN}

La obesidad es una condición multicausal caracterizada por el sobrepeso resultante del desequilibrio del equilibrio energético. El objetivo de este estudio fue evaluar los factores sociodemográficos y de comportamiento asociados con el alto porcentaje de grasa corporal (CG) y obesidad abdominal (OA) en una población adulta, sirvientes de la Universidad Federal de Tocantins. 226 empleados participaron en el estudio, quienes se sometieron a una evaluación antropométrica y respondieron un cuestionario socioeconómico y conductual. La prevalencia de sobrepeso y obesidad fue de 33.09\% y 16.18\%, respectivamente, para las mujeres y $36.67 \%$ y $16.67 \%$, para los hombres. La OA estaba relacionada con la edad en ambos sexos, y la GC directamente relacionada. edad y educación en hombres. $67.78 \%$ de los hombres consumieron alcohol; El 46,32\% de las mujeres eran sedentarias. En los hombres, fumar se asoció con un aumento de la circunferencia de la cintura (WC). La prevalencia de la inactividad física y el consumo de alcohol se consideraron altos en relación con las recomendaciones en la literatura. Los porcentajes de individuos con sobrepeso y obesidad fueron mayores entre los hombres. La PC aumentó con el aumento de la edad en las mujeres. El CG aumentó de acuerdo con el nivel de educación en los hombres. Fumar se asoció con una PC elevada en los hombres; la actividad física no fue un factor protector.

Descriptores: Adiposidad. Composición Corporal. Obesidad Abdomina.

\section{INTRODUÇÃO}

A obesidade é uma condição multicausal, que envolve fatores comportamentais, genéticos, metabólicos, sociais, culturais e psicológicos (CRUZ; ZANON; BOSA, 2015). É caracterizada pelo excesso de peso, resultante do desequilíbrio do balanço energético, sendo considerada um problema de saúde pública que afeta diferentes populações, cujo Índice de Massa Corporal (IMC) é o método mais comumente utilizado para avaliação, por ser pouco invasivo e de baixo custo. Valores entre 25 e $29,99 \mathrm{~kg} / \mathrm{m}^{2}$ é utilizado para classificar o estado nutricional de sobrepeso e valor igual ou superior a $30 \mathrm{~kg} / \mathrm{m}^{2}$ obesidade (SCHURT; LIBERALI; NAVARRO, 2016).

$\mathrm{O}$ excesso de adiposidade constitui fator de risco para o desencadeamento de morbidades, elevando o risco de Doenças Crônicas Não Transmissíveis (DCNT). Entretanto, a deposição de gordura na região abdominal estabelece maiores riscos quando comparada à distribuição generalizada, favorecendo distúrbios metabólicos, doenças cardiovasculares, hipertensão arterial, diabetes mellitus tipo 2, doenças reumáticas e dislipidemias (ABESO, 2016).

Fatores sociodemográficos como idade, sexo, cor, escolaridade, renda per capita e familiar contribuem para a adiposidade corporal, de modo que a baixa renda influencia o consumo de alimentos de baixo custo, consequentemente mais gordurosos e de alta densidade energética, que predispõem ao ganho de peso (MAZUR; NAVARRO, 2015), uma vez que estes alimentos apresentam preços atrativos e facilidade no modo de preparo.

O consumo de álcool, tabagismo, sedentarismo, histórico familiar e presença de doenças familiares são fatores que influenciam na deposição de gordura corporal. Tais fatores constituem importantes agravantes quando associados a outros fatores de risco e ao excesso de peso (PINHO et al., 2013; OLIVEIRA et al., 2015).

Segundo dados da Vigilância de Fatores de Risco e Proteção para Doença por Inquérito Telefônico (VIGITEL) 52,5\% da população com 19 anos ou mais encontram-se com excesso de peso e 17,9\% estão 
obesas. A obesidade está mais presente entre as mulheres e o sobrepeso mais prevalente entre os homens, sendo que o aumento do percentual de gordura é proporcional ao aumento da idade. Palmas, capital do Estado do Tocantins, apresenta $48 \%$ da população com excesso de peso e $16 \%$ com obesidade (BRASIL, 2017).

A compreensão dos fatores determinantes do aumento da deposição de gordura corporal contribui para a diminuição da prevalência do sobrepeso e obesidade e para o planejamento de estratégias e ações que busquem a mudança de hábitos, com o intuito de prevenir o aparecimento ou agravamento da adiposidade e morbidades associadas. O objetivo desse estudo foi avaliar os fatores sociodemográficos e comportamentais associados ao elevado percentual de gordura corporal e à obesidade abdominal em servidores de uma universidade pública.

\section{MATERIAIS E MÉTODOS}

O presente estudo, do tipo transversal, foi realizado por meio da obtenção de dados sobre condições socioeconômicas e hábitos comportamentais, realizado com servidores da Universidade Federal do Tocantins, atendidos no ambulatório de nutrição. Os dados foram obtidos dos prontuários de atendimento ambulatorial.

Todos os indivíduos adultos atendidos na rotina do ambulatório de nutrição foram convidados a participar do estudo. Não foram incluídos idosos, gestantes, puérperas, lactantes e indivíduos que: relataram perda de peso nos últimos seis meses (permitindo flutuação de até 5\% do peso corporal), em uso de quimioterápicos ou uso de medicamentos para perda de peso. Também não foram incluídos indivíduos com alguma limitação física ou outra situação que alterasse a avaliação antropométrica (edemas, membro amputado ou imobilizado por fraturas, cadeirantes etc). A amostra final foi composta por 226 indivíduos, com média de idade de $35 \pm 8,55$ anos e mediana de 36 anos, sendo $60,1 \%$ do sexo feminino.

Todos os participantes assinaram o Termo de Consentimento Livre e Esclarecido, em duas vias. Este estudo foi aprovado pelo Comitê de Ética em Pesquisa com Seres Humanos da Universidade Federal do Tocantins, com $\mathrm{n}^{\circ}$ parecer 2.161.142.

Os hábitos comportamentais foram obtidos através da aplicação de questionário utilizado nas consultas ambulatoriais com questões que abordavam a prática de atividade física (sim ou não) e quantidades de minutos praticados por semana; ingestão de bebida alcoólica (sim ou não); tabagismo (sim ou não) e horas de sono (quantidade de horas dormidas/dia). Foram considerados sedentários aqueles que não realizavam nenhum tipo de atividade física e praticante de atividade física regular aqueles que se exercitavam por 150 minutos ou mais na semana, conforme preconizado pela International Physical Activity Questionnaire (IPAC) (CRAIG et al., 2003).

A avaliação antropométrica foi realizada por meio da aferição da estatura e do peso, os quais foram obtidos por balança digital da marca Welmy, com capacidade máxima de $300 \mathrm{~kg}$ e divisão de $50 \mathrm{~g}$. A estatura foi aferida com um estadiômetro fixo na parede, com extensão de $2,20 \mathrm{~m}$, com divisão em centímetro e milímetros. Ambas as aferições foram realizadas segundo o preconizado por Jelliffe (1968), permitindo obter o índice de massa corporal (IMC), dividindo-se o peso em quilogramas pela altura em metros ao quadrado, classificado conforme preconizado pela OMS (WHO, 1995).

$\mathrm{O}$ perímetro da cintura (PC) foi aferido em duplicata, considerando-se a média das duas medidas, por meio de fita métrica inelástica, sem comprimir a 
pele, no final de uma expiração normal, no ponto médio entre a margem inferior da última costela e a crista ilíaca (HARRIS et al., 2000; WHO, 2000). Quando não foi possível identificar o ponto médio, a medida foi realizada $1 \mathrm{~cm}$ acima da cicatriz umbilical (WILDMAN et al., 2008). O ponto de corte para a classificação do PC foi o recomendado pela OMS (WHO, 2000).

O percentual de gordura corporal $(\% \mathrm{GC})$ foi estimado pelo método de pregas cutâneas, aferidas com o auxílio do adipômetro Lange®, mensuradas em três repetições não consecutivas, nas regiões: tricipital, subescapular, supra-ilíaca e da panturrilha, considerando-se a média das três repetições. Foram adotadas as equações de regressão propostas por Petroski (1995) para predição da densidade corporal (DC) por serem nacionais, generalizadas e desenvolvidas em amostra heterogêneas de indivíduos adultos.

\section{Equações generalizadas para predição da DC,} segundo gênero (PETROSKI, 1995):

$\mathrm{X} 4=\mathrm{PCSE}+\mathrm{PCT}+\mathrm{PCSI}+\mathrm{PCP}$.

$\mathrm{D}=1,10726863-0,00081201(\mathrm{X} 4)+0,00000212$ (X4)2 + (0,00041761) (idade); mulheres.

$\mathrm{D}=1,02902361-0,00067159(\mathrm{X} 4)+0,00000242$ $(\mathrm{X} 4) 2-0,00026073$ (idade) $-0,00056009$ (peso) + 0,00054649 (estatura); homens.

Para conversão da DC em \%GC, utilizou-se a equação de Siri (1961): \%G = (495/D) -450 .

$\mathrm{O} \% \mathrm{GC}$ foi classificado pelos valores de referência para adultos, conforme o sexo, sendo considerados fora da normalidade valores iguais ou superiores a $25 \%$ para os homens ou valores iguais ou superiores a 32\% para as mulheres (LOHMAN, 1992).

Para determinação do índice de conicidade (ICC) foram utilizadas as variáveis peso, estatura e PC, aplicadas à equação matemática proposta por Valdez (1991), não foi adotado ponto de corte para o ICC.

$$
\text { Indice } C=\frac{\text { circunferência da cintura }(\mathrm{m})}{0,109 \sqrt{\frac{\text { peso corporal }(\mathrm{kg})}{\text { estatura }(\mathrm{m})}}}
$$

Os dados foram tabulados no Microsoft Excel e analisados no software estatístico Stata 14. Análises descritivas foram realizadas para os dados sociodemográficos e comportamentais. $\mathrm{O}$ teste $\mathrm{t}$ de Student foi utilizado para verificar as diferenças entre as médias quando a variável apresentou distribuição normal, segundo o sexo. Para verificar associações entre variáveis utilizou-se o teste do qui-quadrado $\left(\chi^{2}\right)$. Foram realizadas análises de correlação de Pearson para verificar o grau de correlação entre variáveis contínuas, quando as duas variáveis apresentaram distribuição normal. Adotou-se o nível de significância estatística de 5\% para todas as análises.

\section{RESULTADOS E DISCUSSÃO}

Dos 226 participantes, mais de $60 \%$ era do sexo feminino e todos eram adultos.

Quanto à escolaridade, 39,3\% dos participantes possuíam ensino superior com pós-graduação, destes 16,8\% possuíam mestrado; $74,8 \%$ dos participantes exerciam a função de técnico-administrativo. Verificou-se que $64,7 \%$ das mulheres e $61,1 \%$ dos homens eram casados, com medianas de renda de $\mathrm{R} \$$ 3083,95 e R\$ 3101,39, para os sexos feminino e masculino respectivamente. A cor parda foi autorreferida por $47,78 \%$ dos participantes.

Avaliando-se os hábitos comportamentais, por meio de análises descritivas, observou-se que o consumo de álcool foi referido por $67,78 \%$ dos homens e $54,41 \%$ das mulheres. Identificou-se que $45,65 \%$ dos homens e $46,32 \%$ das mulheres eram sedentários. A 
prevalência de tabagismo foi observada em $10 \%$ dos homens e $3,68 \%$ das mulheres.

No presente estudo houve predominância do sexo feminino $(60,1 \%)$. Considerando a importância dos fatores comportamentais aqui estudados, como fatores de risco para o desenvolvimento de doenças crônicas, identificou-se que as prevalências de sedentarismo e de consumo de bebidas alcoólicas observadas nesse estudo diferiram do estudo de Nascimento et al. (2015) que encontraram percentual menor em relação ao consumo de bebida alcoólica e maior em relação ao sedentarismo. O tabagismo apresentou-se em percentual próximo ao encontrado na pesquisa de Vigilância de Fatores de Risco e Proteção para Doença por Inquérito Telefônico - VIGITEL (BRASIL, 2017), onde a frequência de fumantes foi de $7,2 \%$, com maior prevalência no sexo masculino.

As prevalências de sobrepeso e obesidade no

\begin{tabular}{ccc}
\hline Variáveis & Correlação de Pearson $(\mathbf{R})$ por sexo & Valores de p \\
\hline \multirow{2}{*}{ Idade } & Perímetro da cintura & \\
& Feminino $\mathrm{R}=0,2677$ & $0,0016^{*}$ \\
Escolaridade & Masculino $\mathrm{R}=0,3076$ & $0,0032^{*}$ \\
& Feminino $\mathrm{R}=-0,2677$ & $0,0078^{*}$ \\
Horas de sono & Masculino $\mathrm{R}=0,2224$ & $0,0352^{*}$ \\
& Feminino $\mathrm{R}=-0,0740$ & 0,3922 \\
& Masculino $\mathrm{R}=0,0359$ & 0,7386 \\
\hline Idade & Gordura corporal & \\
\hline \multirow{2}{*}{ Escolaridade } & Feminino $\mathrm{R}=0,2489$ & $0,0035^{*}$ \\
& Masculino $\mathrm{R}=0,3268$ & $0,0017^{*}$ \\
Horas de sono & Feminino $\mathrm{R}=0,0282$ & 0,7447 \\
& Masculino $\mathrm{R}=0,3016$ & $0,0039^{*}$ \\
& Feminino $\mathrm{R}=0,0305$ & 0,7242 \\
& Masculino $\mathrm{R}=0,0504$ & 0,6391 \\
\hline
\end{tabular}

Tabela 1. Variáveis que estiveram associadas na população estudada, por sexo. 
Para o sexo feminino, o PC esteve diretamente relacionado à idade e inversamente relacionado à escolaridade. Em relação à GC no sexo feminino, essa somente esteve diretamente relacionada à idade. Para o sexo masculino tanto o PC como a GC estiveram diretamente relacionados à idade e à escolaridade.

O PC reflete diretamente a obesidade de localização abdominal. As análises de correlação da presente pesquisa identificaram que essa medida se correlacionou diretamente com a idade e inversamente com a escolaridade no sexo feminino. Resultados semelhantes foram encontrados por Aquino (2014), em que a prevalência de obesidade central aumentou diretamente com o aumento da idade em mulheres. No estudo de Costa et al. (2014), mulheres que praticavam o mesmo nível de atividade física que homens apresentavam maior prevalência de obesidade e excesso de gordura abdominal, o que indica que o excesso de gordura abdominal sofre também influências da genética, da idade, da paridade e do estilo de vida, sendo seu percentual agravado com o sedentarismo.

Quanto à GC e sua relação com a idade, observou-se neste estudo que ela aumenta proporcionalmente ao aumento da idade, assim como relatado no VIGITEL (BRASIL, 2017). No sexo masculino, observou-se correlação significante e positiva entre escolaridade e o \%GC. Resultados diferentes foram encontrados por Moreira (2014) em estudo com servidores técnicos administrativos em educação de uma universidade pública que demonstrou que, à medida que a escolaridade aumenta, o percentual de peso eutrófico também aumenta. Já no estudo de Sá e Moura (2011) o excesso de peso era maior entre aqueles com maior escolaridade, o que vai ao encontro dos deste trabalho, reforçando que a escolaridade propicia trabalhos com maiores níveis hierárquicos e consequentemente, menor esforço físico.

As associações do $\mathrm{PC}$ e do \% $\mathrm{GC}$ com as variáveis comportamentais e de estilo de vida, por sexos encontram-se descritas nas Tabelas 2 e 3 . 
Tabela 2. Associação do perímetro da cintura (PC) e do percentual de gordura corporal (\%GC) com variáveis comportamentais, no sexo feminino.

\begin{tabular}{|c|c|c|c|c|}
\hline \multirow[t]{2}{*}{ Variáveis } & \multicolumn{2}{|c|}{ PC } & \multicolumn{2}{|c|}{$\% \mathrm{GC}$} \\
\hline & $\geq 80$ & $<80$ & $\geq 32$ & $<32$ \\
\hline \multicolumn{5}{|l|}{ Tabagismo } \\
\hline Sim & $3(60 \%)$ & $2(40 \%)$ & $2(40 \%)$ & $3(60 \%)$ \\
\hline \multirow[t]{2}{*}{ Não } & $63(52,5 \%)$ & $57(47,5 \%)$ & $42(35 \%)$ & $78(65 \%)$ \\
\hline & $\mathrm{p}=0,742$ & $\chi^{2}=0,10$ & $\mathrm{p}=0,819$ & $\chi^{2}=0,05$ \\
\hline \multicolumn{5}{|l|}{ Etilismo } \\
\hline Sim & $36(48,6 \%)$ & $38(51,4 \%)$ & $23(31 \%)$ & $51(69 \%)$ \\
\hline \multirow[t]{2}{*}{ Não } & $35(56,5 \%)$ & $27(43,5 \%)$ & $25(40,4 \%)$ & $37(59,6 \%)$ \\
\hline & $\mathrm{p}=0,364$ & $\chi^{2}=0,82$ & $\mathrm{p}=0,261$ & $\chi^{2}=1,26$ \\
\hline \multicolumn{5}{|c|}{$\begin{array}{l}\text { Prática regular de } \\
\text { atividade física }\end{array}$} \\
\hline Sim & $36(49,4 \%)$ & $37(50,6 \%)$ & $22(30,2 \%)$ & $51(69,8 \%)$ \\
\hline \multirow[t]{2}{*}{ Não } & $35(55,5 \%)$ & $28(44,5 \%)$ & $26(41,3 \%)$ & $37(58,7 \%)$ \\
\hline & $\mathrm{p}=0,468$ & $\chi^{2}=0,52$ & $\mathrm{p}=0,176$ & $\chi^{2}=1,83$ \\
\hline
\end{tabular}


Tabela 3. Associação do perímetro da cintura (PC) e do percentual de gordura corporal (\%GC) com variáveis comportamentais, no sexo masculino.

\begin{tabular}{lcccc}
\hline \multicolumn{1}{c}{ Variáveis } & \multicolumn{2}{c}{ PC } & \multicolumn{2}{c}{$\% \mathbf{G C}$} \\
\multicolumn{1}{c}{ Tabagismo } & & & $\mathbf{2 5}$ & $<\mathbf{2 5}$ \\
Sim & $6(66,6 \%)$ & $3(33,3 \%)$ & $4(44,4 \%)$ & $5(55,5 \%)$ \\
Não & $21(28,3 \%)$ & $53(71,6 \%)$ & $29(39,1 \%)$ & $45(60,8 \%)$ \\
& $\mathrm{p}=0,021$ & $\chi^{2}=5,35$ & $\mathrm{p}=0,761$ & $\chi^{2}=0,09$ \\
\multicolumn{1}{c}{ Etilismo } & & & & \\
Sim & $23(37,7 \%)$ & $38(62,3 \%)$ & $28(45,9 \%)$ & $33(54,1 \%)$ \\
Não & $8(27,5 \%)$ & $21(72,4 \%)$ & $9(31 \%)$ & $20(68,9 \%)$ \\
& $\mathrm{p}=0,345$ & $\chi^{2}=0,89$ & $\mathrm{p}=0,180$ & $\chi^{2}=1,79$ \\
Prática regular & & & & \\
de atividade física & & & & \\
Sim & $13(26,5 \%)$ & $36(73,4 \%)$ & $19(38,7 \%)$ & $30(61,2 \%)$ \\
Não & $18(43,9 \%)$ & $23(56,1 \%)$ & $18(43,9 \%)$ & $23(56,1 \%)$ \\
& $\mathrm{p}=0,084$ & $\chi^{2}=2,98$ & $\mathrm{p}=0,623$ & $\chi^{2}=0,24$ \\
\hline
\end{tabular}

As variáveis PC e \%GC não estiveram significantemente associadas ao tabagismo, nem à atividade física ou ao etilismo no sexo feminino. Já no sexo masculino, o tabagismo esteve significativa e positivamente associado ao aumento do PC.

$\mathrm{O}$ hábito de fumar se mostrou estatística e positivamente associado à elevação do $\mathrm{PC}$ no sexo masculino.

A população estudada era composta em sua maior parte por não fumantes. A tendência da população mais jovem é de não ter o hábito de fumar, o que demonstra que as estratégias implementadas no país justificam o decréscimo do tabagismo na população (PORTES; MACHADO; TURCI, 2018).

Oliveira et al. (2015) ao realizarem estudo com 456 adultos da cidade de São Francisco do Conde-BA, constataram que a prevalência de adiposidade abdominal foi superior entre não fumantes e exfumantes.
No estudo de Temponi (2013), indivíduos exfumantes apresentavam IMC médio semelhante ao dos que nunca fumaram, enquanto fumantes apresentavam IMC médio mais baixo em relação aos indivíduos que nunca fumaram.

No estudo de Segheto (2015) realizado com 964 adultos de 20-59 anos, a proporção de adiposidade corporal foi elevada, principalmente no sexo masculino, estando associada com o sexo, idade, estado civil, tabagismo, autoavaliação da saúde e a insatisfação com a imagem corporal. O estudo de Salomão (2013) identificou que tanto a obesidade geral como a abdominal estiveram associadas positivamente ao tabagismo e ao consumo de bebidas alcoólicas.

Na presente análise, o consumo de cigarro e de álcool não estiveram associados à obesidade abdominal ou ao aumento do \%GC no sexo feminino. Assim como a prática de atividades físicas também não se mostrou 
como fator de proteção para ambos os desfechos, em ambos os sexos.

Pinho et al. (2013) ao realizar estudo com adultos de 25-59 anos, constatou que o consumo de bebidas alcoólicas esteve associado a obesidade abdominal apenas no sexo masculino, resultado contrário foi encontrado no presente estudo em que consumir bebida alcoólica não se associou à elevação do PC, que representa a obesidade abdominal, tanto no presente estudo como no estudo de Pinho et al. (2013) foram avaliadas apenas a resposta dicotômica sim ou não. No estudo de Aquino (2014) realizado com 207 funcionários do campus saúde de uma universidade pública de Belo Horizonte, a atividade física mostrouse como fator independentemente associado à obesidade central (OC), de forma que a prevalência de $\mathrm{OC}$ foi menor nas pessoas que eram ativas fisicamente ( $\geq 150$ minutos/semana), desse modo, a atividade física apresentou-se como fator de proteção para esse desfecho, diferente dos nossos resultados, o que indica que tais achados podem sofrer influência da frequência de atividade física, duração e alimentação.

As variáveis antropométricas e sociodemográficas foram distribuídas e comparadas entre os sexos (Tabela 4).

Tabela 4. Distribuição e a comparação entre os sexos das variáveis antropométricas e sociodemográficas.

\begin{tabular}{lcccc}
\hline \multicolumn{1}{c}{ Variável } & População Geral & Mulheres & Homens & p \\
\hline Escolaridade (anos) & $18,12 \pm 5,09$ & $17,57 \pm 4,64$ & $18,96 \pm 5.62$ & $0,0438^{*}$ \\
Renda (R\$) & $3090,9 \pm 2317,27$ & $3083,95 \pm 2392,32$ & $3101,39 \pm 2212,20$ & 0,9560 \\
Estatura (m) & $1,64 \pm 0,097$ & $1,59 \pm 0,073$ & $1.72 \pm 0,074$ & $0,0000^{*}$ \\
Peso (kg) & $70,71 \pm 15,49$ & $65,58 \pm 13,25$ & $78,46 \pm 15,47$ & $0,0000^{*}$ \\
IMC (kg/m $\left.{ }^{2}\right)$ & $25,88 \pm 4,61$ & $25,65 \pm 4,81$ & $26,21 \pm 4,28$ & 0,3748 \\
PC (cm) & $85,41 \pm 0,80$ & $82,12 \pm 11,06$ & $90,37 \pm 11,92$ & $0,0000^{*}$ \\
ICC & $1,20 \pm 0,07$ & $1,17 \pm 0,07$ & $1,23 \pm 0,07$ & $0,0000^{*}$ \\
PT (mm) & $22,18 \pm 8,59$ & $25,11 \pm 7,22$ & $17,75 \pm 8,64$ & $0,0000^{*}$ \\
PSE (mm) & $23,03 \pm 9,95$ & $24,02 \pm 10,12$ & $21,54 \pm 9,53$ & 0,0668 \\
PSI (mm) & $26,70 \pm 11,03$ & $27,37 \pm 11,02$ & $25,70 \pm 11,05$ & 0,2684 \\
PP (mm) & $18,64 \pm 8,28$ & $21,92 \pm 7,03$ & $13,67 \pm 7,55$ & $0,0000^{*}$ \\
SOMA4PC (mm) & $90,56 \pm 32,22$ & $98,42 \pm 30,33$ & $78,69 \pm 31,49$ & $0,0000^{*}$ \\
DC (g/ml) & $1,03 \pm 0,01$ & $1,03 \pm 0,01$ & $1,04 \pm 0,01$ & $0,0000^{*}$ \\
GC (\%) & $27,54 \pm 6,50$ & $29,78 \pm 5,31$ & $24,16 \pm 6,70$ & $0,0000^{*}$ \\
\hline
\end{tabular}

* Significantivo pelo Teste t-Student para amostras independentes. IMC - Índice de Massa Corporal; PC - perímetro da cintura; ICC - índice de conicidade; PT - prega tricipital; PSE - prega subescapular; PSI - prega supra-ilíaca; PP - prega da panturrilha; SOMA4PC - soma de quatro pregas cutâneas; DC - densidade corporal; GC - gordura corporal. 
Escolaridade, peso, estatura, PC, ICC, PT, PP, somatório de 4 pregas, densidade corporal e \%GC diferiram significantemente entre os sexos, sendo que o sexo masculino apresentou as maiores médias de tempo de estudo formal, de peso, de estatura, de PC, de ICC e de DC. As mulheres apresentaram as maiores médias de prega cutânea triciptal, prega da panturrilha, somatório de 4 pregas corporais e de percentual de gordura corporal.

As medidas antropométricas foram utilizadas com o intuito de avaliar a adiposidade corporal na população estudada. Tais análises demonstraram que houve diferenças estatisticamente significativas entre ambos os sexos para a maioria das variáveis (Tabela 4), com exceção da renda, IMC, PSE e PSI, o que indica que a composição corporal diferiu entre os sexos. A diferença significativa em relação ao $\% \mathrm{GC}$ foram ao encontro do estudo de Freitas et al. (2014), em que mulheres apresentaram maiores porcentagens para essa variável. Outros estudos também demonstraram diferenças significativas entre os sexos para as variáveis peso, estatura e PC, semelhantemente ao presente trabalho (FREITAS et al., 2014; GUEDES; ALVES, 2017).

O ICC aqui estudado avalia a obesidade e distribuição de gordura corporal, e vários estudos o utilizam com intuito de determinar sua relação com variáveis consideradas de risco para o desenvolvimento de doenças cardiovasculares e crônicas. No presente estudo, o ICC apresentou maiores médias no sexo masculino. Dantas et al. (2015), ao analisar a prevalência de risco aumentado para o desenvolvimento de doenças cardiovasculares obtiveram maior classificação de risco para a população geral e sexo masculino observada pela avaliação do ICC, e para o sexo feminino, se deu por meio da medida do PC.

Santos (2018) ao realizar revisão com estudos que abordavam o uso do ICC observou que entre os índices utilizados na avaliação antropométrica, o ICC esteve associado a doenças cardiometabólicas, o que indica que tal índice apresentou-se válido para a estimativa da gordura visceral e risco de morbidade. No presente estudo o ICC não foi associado a outras variáveis, porém diferiu significativamente entre os sexos, assim como relatado no estudo de Dantas et al. (2015).

\section{CONCLUSÃO}

O presente estudo teve como objetivo avaliar os fatores sociodemográficos e comportamentais associados ao elevado percentual de gordura corporal e à obesidade abdominal em servidores de uma universidade pública.

Os resultados demonstraram que o consumo de bebida alcoólica e o sedentarismo são elevados, mesmo com a maioria dos participantes se declararem ativos em relação à prática de atividade física, estes achados em relação a estudos desenvolvidos em populações semelhantes se mostraram elevados.

Diante dos resultados pode-se afirmar que os hábitos comportamentais são fatores importantes para a manutenção da saúde, necessitando de intervenções para melhorar os índices de sobrepeso e obesidade, possibilitando assim a melhora das condições de saúde e de vida dos servidores, pois fatores como idade e 
grau de escolaridade contribuíram para o aumento do PC no sexo feminino e GC no sexo masculino, respectivamente.

Assim, é importante ressaltar que o habito de fumar foi considerado baixo, porém este associou-se ao PC elevado no sexo masculino e a prática de atividade física não se demostrou fator de proteção, o que demostram a necessidade em estabelecer avaliações periódicas do estado nutricional desse público.

Todos os autores declaram não haver qualquer potencial conflito de interesses referente a este artigo.

\section{REFERÊNCIAS}

AQUINO, A. L. Fatores associados à obesidade central em funcionários do campus saúde de uma Universidade Pública. Dissertação de Mestrado. Escola de Enfermagem, Universidade Federal de Minas Gerais, Belo Horizonte, 2014.

ASSOCIAÇÃO BRASILEIRA PARA O ESTUDO DA OBESIDADE E DA SÍNDROME METABÓLICA. Diretrizes brasileiras de obesidade 2016 / ABESO Associação Brasileira para o Estudo da Obesidade e da Síndrome Metabólica. 4. ed. São Paulo, 2016.

BRASIL. Ministério da Saúde. Vigitel Brasil 2015 Saúde Suplementar: vigilância de fatores de risco e proteção para doenças crônicas por inquérito telefônico [recurso eletrônico] / Ministério da Saúde, Agência Nacional de Saúde Suplementar. Brasília: Ministério da Saúde, 2017. 170 p.

CRAIG, C. L.; MARSHALL, A. L.; SJÖSTRÖM, M.; BAUMAN, A. E.; BOOTH M. L.; AINSWORTH, B. E.; PRATT, M.; EKELUND, U.; YNGVE, A.; SALLIS, J. F.; OJA, P. International physical activity questionnaire: 12 -country reliability and validity. Medicine and science in sports and exercise. v. 35, n. 8, p. 1381-95, 2003.
COSTA, M. A. P.; VASCONCELOS, A. G. G.; FONSECA, M. J. M. Prevalência de obesidade, excesso de peso e obesidade abdominal e associação com prática de atividade física em uma universidade federal. Rev Bras Epidemiol, p. 421-436, abr -jun 2014.

CRUZ, S. H.; ZANON, R. B.; BOSA, C. A. Relação entre apego e obesidade: Revisão sistemática da literatura. Psico. Porto Alegre, PUCRS, v. 46, n. 1, p. 6-15, 2015.

DANTAS, E. M. S.; PINTO, C. J.; FREITAS, R. P. A.; MEDEIROS, A. C. Q.vConcordância na avaliação de risco cardiovascular a partir de parâmetros antropométricos. Einstein. n. 13, v. 3, p.376-380, 2015.

FREITAS, L.; SILVA, L. A.; SCHIMANSKI, P. C.; PORTELA, B. S.; FRAGA, C. H. W. Perfil antropométrico e da aptidão física relacionada á saúde em homens e mulheres adultos de diferentes faixas etárias. Revista Brasileira de Prescrição e Fisiologia do Exercício, São Paulo, v.8, n.48, p. 594-598. Jul/Ago. 2014.

GUEDES, V. C.; ALVES, M. K. Prevalência e fatores de risco para excesso de peso em funcionários dos turnos vespertino e noturno de um hospital da Serra Gaúcha. Revista Brasileira de Obesidade, Nutrição e Emagrecimento, São Paulo. v. 11. n. 66. p. 420-427. Nov./Dez, 2017.

HARRIS, M. M.; STEVENS, J.; THOMAS, N.; SCHREINER, P.; FOLSOM, A. R. Associations of fat distribution and obesity with hypertension in a bi-ethnic population: The ARIC Study. Obesity Research Journal, v.8, n.7, p. 516-524, 2000.

JELLIFFE, D. B. Evaluación del estado de nutrición de la comunidad. Ginebra: OMS; 1968. Publicação científica $n^{\circ} 53$.

LOHMAN, T. G. Advances in body composition assessment. Champaign: Human Kinetics, 1992. 150 p (current Issues in Exercise Science. Monograph n. 3).

MAZUR, C. E.; NAVARRO, F. Insegurança alimentar e obesidade em adultos: Qual a relação? Saúde (Santa Maria), v. 41, n. 2, Jul./Dez, p. 35-44, 2015. 
MOREIRA, L. B. F. Estado nutricional dos servidores técnicos administrativos em educação de uma universidade pública associado aos hábitos alimentares e às práticas de atividade física. Dissertação de Mestrado. Universidade Federal de Juiz de Fora, MG, 2014.

NASCIMENTO, L. S.; DIAS, R. M.; DUTRA, C. D. T.; SILVA, L. M. C.; PEDROSA, L. N.; ARAÚJO, J. S.; FRAZÃO, A. G. F. Fatores de risco para doenças crônicas não transmissíveis e variáveis sociodemográficas de servidores públicos. Rev Bras Promoç Saúde, Fortaleza, v. 28, n.2, p. 230-239, abr.jun., 2015.

OLIVEIRA, L. C.; WEST, L. E. M.; ARAÚJO, E. A.; BRITO, J. S.; SOBRINHO, C. L. N. Prevalência de adiposidade abdominal em adultos de São Francisco do Conde, Bahia, Brasil, 2010. Epidemiol. Serv. Saúde, v. 24, n. 1, p.135-144, jan-mar, 2015.

PETROSKI, E. L. Desenvolvimento e validação de equações generalizadas para a estimativa da densidade corporal em adultos. Tese de Doutorado. Universidade Federal de Santa Maria, RS, 1995.

PINHO, C. P. S.; DINIZ, A. S.; ARRUDA, I. K. G.; FILHO, M. B.; COELHO, P. C.; SEQUEIRA, L. A. S.; LIRA, P. I. C. Prevalência e fatores associados à obesidade abdominal em indivíduos na faixa etária de 25 a 59 anos do Estado de Pernambuco, Brasil. Cad. Saúde Pública, v. 29, n. 2, p. 313-324, fev, 2013.

PORTES, L. H.; MACHADO, C. V.; TURCI, S. R. B. Trajetória da política de controle do tabaco no Brasil de 1986 a 2016. Cad. Saúde Pública; v. 34, n. 2, 2018.

SÁ, N. N. B.; MOURA, E. C. Excesso de peso: determinantes sociodemográficos e comportamentais em adultos, Brasil, 2008. Cad. Saúde Pública, Rio de Janeiro, v. 27, n. 7, p. 1380-1392, 2011.

SALOMÃO, N. C. Excesso de peso, obesidade abdominal e estilo de vida em adultos e idosos. Dissertação de Mestrado. Faculdade de Nutrição, Universidade Federal de Pelotas, Rio Grande do Sul, 2013.
SANTOS, P. C. Relação entre índices de gordura corporal e massa óssea em adultos e idosos: Estudo ISA - Capital (2015). Dissertação de Mestrado. Faculdade de Saúde Pública, Universidade de São Paulo, São Paulo, 2018.

SEGHETO, W. Índice de adiposidade corporal: validação e fatores associados em adultos em um estudo de base populacional, Viçosa, MG, Brasil. Tese de Doutorado. Universidade Federal de Viçosa, Minas Gerais, 2015.

SIRI, W. E. Body composition from fluid spaces and density: analyses of methods. In BROZEK J, HENSCHEL A (eds.). Techniques for measuring body composition. Washington: National Academy of Science, 1961.

SCHURT, A.; LIBERALI, R.; NAVARRO, F. Exercício contra resistência e sua eficácia no tratamento da obesidade: Uma revisão sistemática. Revista Brasileira de Obesidade, Nutrição e Emagrecimento, v. 10, n. 59, p. 215223, Set./Out, 2016.

TEMPONI, H. R. Avaliação da associação entre tabagismo e índice de massa corporal segundo níveis de escolaridade. Dissertação de Mestrado. Escola de Enfermagem, Universidade Federal de Minas Gerais, Belo Horizonte, 2013.

VALDEZ, R. A simple model-based index of abdominal adiposity. J Clin Epidemiol. v. 44, $\mathrm{n}^{\circ}$ 9, p. 955-6, 1991.

WILDMAN, R. P.; MUNTNER, P.; REYNOLDS, K.; McGINN, A. P.; RAJPATHAK, S.; WYLIE-ROSETT, J.; SOWERS, M. R. The obese without cardiometabolic risk fator clustering and the normal weight with cardiometabolic risk factor clustering. Arch Intern Med, v. 168, n. 15, 2008.

WORLD HEALTH

ORGANIZATION. Obesity: preventing and managing the global epidemic. Geneva, 2000. WORLD HEALTH ORGANIZATION. Physical status: the use and interpretation of anthropometry. Report of a WHO expert committee. Geneva, 1995. 